\title{
Co-prime order graphs of finite Abelian groups and dihedral groups
}

\author{
Amit Sehgal ${ }^{a, *}$, Manjeet ${ }^{a}$, Dalip Singh ${ }^{b}$ \\ ${ }^{a}$ Department of Mathematics, Pt. NRS Govt. College, Rohtak (Haryana), India. \\ ${ }^{b}$ Department of Mathematics, Maharshi Dayanand University, Rohtak (Haryana), India.
}

\begin{abstract}
The co-prime order graph $\Theta(G)$ of a given finite group is a simple undirected graph whose vertex set is the group $\mathrm{G}$ itself, and any two vertexes $x, y$ in $\Theta(G)$ are adjacent if and only if $\operatorname{gcd}(\mathrm{o}(\mathrm{x}), \mathrm{o}(\mathrm{y}))=1$ or prime. In this paper, we derive a precise formula to count the vertex's degree in the co-prime order graph of a finite Abelian group or dihedral group.We also investigate the Laplacian spectrum of the co-prime order graph $\Theta(G)$ when $G$ is a finite Abelian p-group, $\mathbb{Z}_{p}{ }^{t} \times \mathbb{Z}_{q}{ }^{s}$ or a dihedral group $\mathrm{D}_{\mathrm{p}^{n}}$.
\end{abstract}

Keywords: Co-prime order graph, finite Abelian group, dihedral group, Laplacian spectrum.

2020 MSC: 05C25, 05C50.

(C)2021 All rights reserved.

\section{Introduction}

The investigation of graphs related to various algebraic structures is an important research direction, where many active mathematicians have been working recently. It is common to generate graphs from groups. In [5], the author studied intersection graphs defined on a finite Abelian group. The cayley digraph is also an important class of directed graphs defined on finite groups and readers may refer to [3]. In [7], authors introduced the directed power graph of a group as a directed graph whose vertex set is the group, and there is an arc from vertex $u$ to the other vertex $v$ whenever $v$ is a power of $u$. From motivated by this concept, definition of the power graph $\mathcal{G}(\mathrm{G})$ of a semi-group $\mathrm{G}$ as a graph with $\mathrm{G}$ as its vertex set, and there is an edge between two distinct vertices if one is a power of the other in [4]. In survey paper [1] which includes many good results on power graphs. Concept of minimum degree and edge-connectivity of power graph of finite cyclic group studied in [9, 10]. Ma et al. [8] firstly introduced co-prime graph of a group as a simple graph whose vertex set is the group, and any distinct two vertexes $x, y$ in $G$ are adjacent if and only if $\operatorname{gcd}(o(x), o(y))=1$. Banerjee [2] introduced the co-prime order graph of a group as a simple graph whose vertex set is the group, and there is an arc from vertex $u$ to the other vertex $v$ whenever $\operatorname{gcd}(\mathrm{o}(\mathrm{u}), \mathrm{o}(v))=1$ or prime. The degree of a vertex of a graph associated with a finite

\footnotetext{
*Corresponding author

Email addresses: amit_sehgal_iit@yahoo.com (Amit Sehgal), sainimanjeet1994@gmail.com (Manjeet), dsmdur@gmail.com (Dalip Singh)
}

doi: $10.22436 /$ jmcs.023.03.03

Received: 2020-08-19 Revised: 2020-09-23 Accepted: 2020-09-28 
group is also used to study the structural properties of the graph [11]. In Section 3 of the present paper, we obtain the degree of a vertex in the co-prime order graph of a finite Abelian group or dihedral group $D_{n}$. In Section 4 of the present paper, we obtain the laplacian spectrum of the co-prime order graph $\Theta(G)$ when $G$ is finite Abelian p-group, $\mathbb{Z}_{p}{ }^{t} \times \mathbb{Z}_{q}{ }^{s}$ or dihedral group $D_{p^{n}}$.

\section{Preliminaries and notation}

Let $\Gamma$ be a finite simple graph. Its Laplacian matrix is the matrix $L(\Gamma)=\mathrm{D}(\Gamma)-\mathrm{A}(\Gamma)$, where $\mathrm{D}(\Gamma)$ is the diagonal matrix of vertex degrees of $\Gamma$ and $A(\Gamma)$ is the adjacency matrix of $\Gamma$. The Laplacian polynomial of $\Gamma$ is the characteristic polynomial of $L(\Gamma)$.

Let $\mathrm{G}$ be a group. Let $e$ denote the identity element of $\mathrm{G}$ and $|\mathrm{G}|$ denote the order of $\mathrm{G}$ used throughout the paper. The cyclic group of order $n$ is usually denoted by $\mathbb{Z}_{n}$. Let $g$ be an arbitrary element of $G$. We denote the order of $\mathrm{g}$ by $|\mathrm{g}|$. Let $\mathrm{H}$ and $\mathrm{K}$ be two normal subgroups of $\mathrm{G}$.

\section{Results for degree}

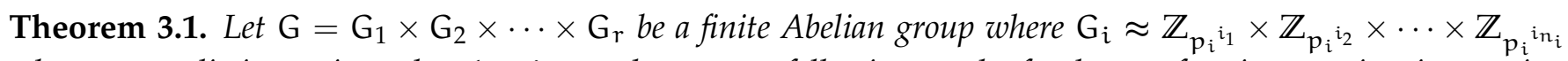
where $p_{i}$ are distinct prime when $1 \leqslant i \leqslant r$, then we get following results for degree of various vertices in co-prime order graph of group $\mathrm{G}$ :

(i) $\operatorname{deg}(x)=\prod_{k=1}^{r} p_{k}^{\sum_{j=1}^{n_{k}} k_{j}}-1$, when $\mathrm{o}(x)=1$ or $p_{i}$ where $i=1,2, \ldots, r$;

(ii) $\operatorname{deg}(x)=\left(-k-l+1+\sum_{j=1}^{j=k}\left|G_{\alpha_{j}}\right|+\sum_{j=1}^{j=l}\left(p_{\beta_{j}}\right)^{n_{\beta_{j}}}\right) \frac{|G|}{\left(\prod_{i=1}^{i=k}\left|G_{\alpha_{i}}\right|\right)\left(\prod_{i=1}^{i=l}\left|G_{\beta_{i}}\right|\right)}$, when

$$
\mathrm{o}(\mathrm{x})=\left(\prod_{i=1}^{i=k} \mathrm{p}_{\alpha_{i}}\right)\left(\prod_{i=1}^{i=l} \mathrm{p}_{\beta_{i}}{ }^{\gamma_{i}}\right)
$$

where $2 \leqslant k+l \leqslant r$ or $l \geqslant 1$ and $p_{\alpha_{1}}, p_{\alpha_{2}}, \cdots, p_{\alpha_{k}}, p_{\beta_{1}}, p_{\beta_{2}}, \cdots, p_{\beta_{l}}$ are distinct primes from set $\left\{p_{1}, p_{2}, \cdots, p_{r}\right\}$ and $\gamma_{1}, \gamma_{2}, \cdots, \gamma_{l} \geqslant 2$.

Proof. Let $x$ be an arbitrary element group $G$ of order $\prod_{i=1}^{r} p_{i} \alpha_{i}$, then there exists unique $x_{p_{i}} \in G_{i}$ of order $p_{i}{ }^{\alpha_{i}}$ such that $x=\prod_{i=1}^{r} x_{p_{i}}$. We also know that group $G_{i}$ has exactly $p_{i}{ }^{n_{i}}-1$ elements of order $p_{i}$.

We consider the following cases as follows:

Case 1: Let $o(x)=1$ or $p_{i}$.

We know that $\operatorname{gcd}(o(x), o(y))=1$ or $p_{i}$ for every $y \in G$, so vertex $x$ is connected all the vertices, hence $\operatorname{deg}(x)=\prod_{k=1}^{r} p_{k}^{\sum_{j=1}^{n_{k}} k_{j}}-1$.

Case 2: Let $o(x)=\left(\prod_{i=1}^{i=k} p_{\alpha_{i}}\right)\left(\prod_{i=1}^{i=l} p_{\beta_{i}} \gamma_{i}\right)$ where $2 \leqslant k+l \leqslant r$ or $l \geqslant 1$ and $p_{\alpha_{1}}, p_{\alpha_{2}}, \ldots, p_{\alpha_{k}}, p_{\beta_{1}}, p_{\beta_{2}}, \cdots$, $p_{\beta_{l}}$ are distinct primes from set $\left\{p_{1}, p_{2}, \cdots, p_{r}\right\}$ and $\gamma_{1}, \gamma_{2}, \cdots, \gamma_{l} \geqslant 2$. We have

$$
\left(\left(\prod_{i=1}^{i=k} p_{\alpha_{i}}\right)\left(\prod_{i=1}^{i=l} p_{\beta_{i}}^{\gamma_{i}}\right), \frac{|G|}{\left(\prod_{i=1}^{i=k}\left|G_{\alpha_{i}}\right|\right)\left(\prod_{i=1}^{i=l} G_{\beta_{i}}\right)}\right)=1,
$$

then

$$
\left(\left(\prod_{i=1}^{i=k} p_{\alpha_{i}}\right)\left(\prod_{i=1}^{i=l} p_{\beta_{i}} \gamma_{i}\right), \frac{\left|G_{\alpha_{j}} \| G\right|}{\left(\prod_{i=1}^{i=k}\left|G_{\alpha_{i}}\right|\right)\left(\prod_{i=1}^{i=l} G_{\beta_{i}}\right)}\right)=p_{\alpha_{j}},
$$

where $1 \leqslant j \leqslant k$ and

$$
\left(\left(\prod_{i=1}^{i=k} p_{\alpha_{i}}\right)\left(\prod_{i=1}^{i=l} p_{\beta_{i}} \gamma_{i}\right), \frac{p_{\beta_{s}}|G|}{\left(\prod_{i=1}^{i=k}\left|G_{\alpha_{i}}\right|\right)\left(\prod_{i=1}^{i=l}\left|G_{\beta_{i}}\right|\right)}\right)=p_{\beta_{s}},
$$


where $1 \leqslant s \leqslant l$.

So, vertex $x$ is adjacent to every vertex whose order divides

$$
\frac{\left|G_{\alpha_{j}}\right||G|}{\left(\prod_{i=1}^{i=k}\left|G_{\alpha_{i}}\right|\right)\left(\prod_{i=1}^{i=l}\left|G_{\beta_{i}}\right|\right)},
$$

or

$$
\left.\frac{p_{\beta_{s}}|G|}{\left(\prod_{i=1}^{i=k}\left|G_{\alpha_{i}}\right|\right)\left(\prod_{i=1}^{i=l} G_{\beta_{i}}\right)}\right)
$$

for every $1 \leqslant j \leqslant k$ and $1 \leqslant s \leqslant l$. So, vertex $x$ is adjacent to

$$
\begin{aligned}
& \sum_{j=1}^{j=k}\left(\frac{\left|G_{\alpha_{j}}\right||G|}{\left(\prod_{i=1}^{i=k}\left|G_{\alpha_{i}}\right|\right)\left(\prod_{i=1}^{i=l}\left|G_{\beta_{i}}\right|\right)}-\frac{|G|}{\left(\prod_{i=1}^{i=k}\left|G_{\alpha_{i}}\right|\right)\left(\prod_{i=1}^{i=l}\left|G_{\beta_{i}}\right|\right)}\right) \\
& \left.\left.\quad+\sum_{j=1}^{j=l} \frac{\left(p_{\beta_{j}}\right)^{n_{\beta_{j}}|G|}}{\left(\prod_{i=1}^{i=k}\left|G_{\alpha_{i}}\right|\right)\left(\prod_{i=1}^{i=l}\left|G_{\beta_{i}}\right|\right)}\right)-\frac{|G|}{\left(\prod_{i=1}^{i=k}\left|G_{\alpha_{i}}\right|\right)\left(\prod_{i=1}^{i=l}\left|G_{\beta_{i}}\right|\right)}\right)+\frac{|G|}{\left(\prod_{i=1}^{i=k}\left|G_{\alpha_{i}}\right|\right)\left(\prod_{i=1}^{i=l}\left|G_{\beta_{i}}\right|\right)} \\
& \quad=\left(-k-l+1+\sum_{j=1}^{j=k}\left|G_{\alpha_{j}}\right|+\sum_{j=1}^{j=l}\left(p_{\beta_{j}}\right)^{n_{\beta_{j}}}\right) \frac{|G|}{\left(\prod_{i=1}^{i=k}\left|G_{\alpha_{i}}\right|\right)\left(\prod_{i=1}^{i=l}\left|G_{\beta_{i}}\right|\right)} .
\end{aligned}
$$

Hence we get desired result.

Corollary 3.2. If $\mathrm{o}(\mathrm{x})$ is a prime, then $\mathrm{deg}(\mathrm{x})=\mathrm{n}-1$ in any co-prime order graph of order $\mathrm{n}$.

Proof. By definition of co-prime order graph vertex $x$ is connected with every other vertex of graph other than itself, hence $\operatorname{deg}(x)=n-1$.

Corollary 3.3. Let $\mathrm{D}_{\mathrm{n}}=\left\{\mathrm{f}^{\mathrm{i}} \mathrm{r}^{\mathrm{j}} \mid \mathrm{o}(\mathrm{f})=2, \mathrm{o}(\mathrm{r})=\mathrm{n}, \mathrm{rf}=\mathrm{fr}^{-1}\right\}$ be a finite non-Abelian group with $2 \mathrm{n}$, then we get following results for degree of various vertices in co-prime order graph of group $\mathrm{D}_{\mathrm{n}}$ :

(i) If $\mathrm{o}(\mathrm{x}) \neq 2$, then difference of degree's of $\mathrm{x}$ in co-prime prime order graph of group $\mathrm{D}_{\mathrm{n}}$ and group $\langle\mathrm{r}\rangle$ is $\mathrm{n}$.

(ii) If $\mathrm{o}(x)=2$, then $\operatorname{deg}(x)=2 n-1$.

Proof. Take $x$ be arbitrary element of $D_{n}$.

Case 1: If $o(x) \neq 2$, then $x$ is connected with following vertices:

(i) All vertices of the type $\mathrm{fr}^{\mathrm{j}}$ where $j=1,2, \cdots, \mathrm{n}$. These vertices are $n$ vertices.

(ii) Vertices of the type $r^{j}$ if $\left(o(x), \frac{n}{(n, j)}\right)=1$ or prime where $j=1,2, \cdots, n$. Number of these vertices are same as degree of any vertex of order equal to $\mathrm{o}(\mathrm{x})$ in co-prime order graph of group $\mathbb{Z}_{n}$.

Hence, we get difference of degree's of $x$ in co-prime prime order graph of group $D_{n}$ and group $\langle r\rangle$ is $n$.

Case 2: If $\mathrm{o}(x)=2$, then by use of corollary 1 we get $\operatorname{deg}(x)=2 n-1$.

\section{Laplacian spectrum}

Theorem 4.1. Let

$$
L=\left(\begin{array}{cc}
A & C \\
C^{\top} & B
\end{array}\right)
$$

where

$$
A=\left(\begin{array}{ccccc}
p+q-1 & -1 & -1 & \ldots & -1 \\
-1 & p+q-1 & -1 & \ldots & -1 \\
-1 & -1 & p+q-1 & \ldots & -1 \\
\ldots & \ldots & \ldots & \ldots & \ldots \\
-1 & -1 & -1 & \ldots & p+q-1
\end{array}\right)_{p \times p}
$$


$\mathrm{C}$ is a $\mathrm{p} \times \mathrm{q}$ matrix whose all entries are -1 and $\mathrm{B}=\mathrm{pI} \mathrm{I}_{\mathrm{q} \times \mathrm{q}}$, then Laplacian spectrum of $\mathrm{L}$ where $\mathrm{p}$ and $\mathrm{q} \geqslant 1$ is 0 with multiplicity $1, \mathrm{p}+\mathrm{q}$ with multiplicity $\mathrm{p}$ and $\mathrm{p}$ with multiplicity $\mathrm{q}-1$.

Proof. We now proceed to find the spectrum of $L$ for every prime $p$. The characteristic polynomial of $L$ is given by

$$
\Lambda=\operatorname{det}(x \mathrm{I}-\mathrm{L})=\operatorname{det}\left(\begin{array}{cc}
x \mathrm{I}-\mathrm{A} & -\mathrm{C} \\
-\mathrm{C}^{\top} & x \mathrm{I}-\mathrm{B}
\end{array}\right) .
$$

Apply the row operation $R_{1} \rightarrow \sum_{i=1}^{p+q} R_{i}$ and take $x$ common from 1 st row, we get

$$
\Lambda=\operatorname{det}(x \mathrm{I}-\mathrm{L})=x \operatorname{det}\left(\begin{array}{cc}
\mathrm{D} & \mathrm{F} \\
-\mathrm{C}^{\mathrm{T}} & x \mathrm{I}-\mathrm{B}
\end{array}\right),
$$

where

$$
\mathrm{D}=\left(\begin{array}{cccc}
1 & 1 & \ldots & 1 \\
1 & x-p-q+1 & \ldots & 1 \\
\ldots & \ldots & \ldots & \ldots \\
1 & 1 & \ldots & x-p-q+1
\end{array}\right)_{p \times p}, \quad F=\left(\begin{array}{cccc}
1 & 1 & \ldots & 1 \\
1 & 1 & \ldots & 1 \\
\ldots & \ldots & \ldots & \ldots \\
1 & 1 & \ldots & 1
\end{array}\right)_{p \times q}
$$

Now we apply row operations $R_{i} \rightarrow R_{i}-R_{1}, \forall i=2,3, \cdots, p+q$, we get

$$
\Lambda=\operatorname{det}(x \mathrm{I}-\mathrm{L})=x \operatorname{det}\left(\begin{array}{cc}
\mathrm{G} & \mathrm{H} \\
\mathrm{I} & \mathrm{J}
\end{array}\right) \text {, }
$$

where

$$
\begin{gathered}
\mathrm{G}=\left(\begin{array}{ccccc}
1 & 1 & \ldots & 1 \\
0 & x-p-q & \ldots & 0 \\
\ldots & \ldots & \ldots & \ldots \\
0 & 0 & \ldots & p-q
\end{array}\right)_{p \times p}, \quad F=\left(\begin{array}{cccc}
1 & 1 & \ldots & 1 \\
0 & 0 & \ldots & 0 \\
\ldots & \ldots & \ldots & \ldots \\
0 & 0 & \ldots & 0
\end{array}\right)_{p \times q}, \\
I=\left(\begin{array}{cccc}
0 & 0 & \ldots & 0 \\
0 & 0 & \ldots & 0 \\
\ldots & \ldots & \ldots & \ldots \\
0 & 0 & \ldots & 0
\end{array}\right)_{q \times p} \quad, \quad J=\left(\begin{array}{cccc}
x-p-1 & -1 & \ldots & -1 \\
-1 & x-p-1 & \ldots & -1 \\
\ldots & \ldots & \ldots & \ldots \\
-1 & -1 & \ldots & x-p-1
\end{array}\right)_{q \times q} .
\end{gathered}
$$

Now we rewrite

$$
\Lambda=\operatorname{det}(x \mathrm{I}-\mathrm{L})=x(x-p-q)^{p-1} \operatorname{det}(J) .
$$

Apply the row operation $R_{1} \rightarrow \sum_{i=1}^{q} R_{i}$ to $\operatorname{det}(J)$ and take $x-p-q$ common from 1 st row, we get

$$
\Lambda=\operatorname{det}(x \mathrm{I}-\mathrm{L})=x(x-p-q)^{p} \operatorname{det}(\mathrm{K}),
$$

where

$$
\mathrm{K}=\left(\begin{array}{cccc}
1 & 1 & \ldots & 1 \\
-1 & x-p-1 & \ldots & -1 \\
\ldots & \ldots & \ldots & \ldots \\
-1 & -1 & \ldots & x-p-1
\end{array}\right)_{\mathrm{q} \times \mathrm{q}}
$$

Now we apply row operations $R_{i} \rightarrow R_{i}+R_{1}, \forall i=2,3, \cdots$, q, we get

$$
\Lambda=\operatorname{det}(x \mathrm{I}-\mathrm{L})=x(x-\mathrm{p}-\mathrm{q})^{\mathrm{p}} \operatorname{det}\left(\mathrm{K}_{1}\right),
$$

where

$$
\mathrm{K}_{1}=\left(\begin{array}{cccc}
1 & 1 & \cdots & 1 \\
0 & x-p & \cdots & 0 \\
\cdots & \cdots & \cdots & \cdots \\
0 & 0 & \cdots & x-p
\end{array}\right)_{\mathrm{q} \times \mathrm{q}}
$$


Finally, we get $\Lambda=\operatorname{det}(x I-L)=x(x-p-q)^{p}(x-p)^{q-1}$. Hence the eigenvalues of L are 0 with multiplicity $1, p+q$ with multiplicity $p$ and $p$ with multiplicity $q-1$.

Theorem 4.2. Laplacian spectrum of group $\mathbb{Z}_{\mathrm{p}}^{\mathrm{t}}$ where $\mathrm{p}$ is prime and $\mathrm{t} \geqslant 1$ are 0 with multiplicity 1 and $\mathrm{p}^{\mathrm{t}}$ with multiplicity $\mathrm{p}^{\mathrm{t}}-1$.

Proof. We now proceed to find the Laplacian spectrum of group and denoted by L. The rows and columns of the matrix $L$ have been indexed in the following ways:

We start with the zero element $[0]$ of $\mathbb{Z}_{\mathrm{p}}^{\mathrm{t}}$. We then list the remaining elements of $\mathbb{Z}_{\mathrm{p}}^{\mathrm{t}}$. Using the above indexing the matrix of $L$ takes the following form:

$$
\mathrm{L}=\left(\begin{array}{ccccc}
p^{\mathrm{t}}-1 & -1 & -1 & \ldots & -1 \\
-1 & p^{\mathrm{t}}-1 & -1 & \ldots & -1 \\
-1 & -1 & p^{\mathrm{t}}-1 & \ldots & -1 \\
\ldots & \ldots & \ldots & \ldots & \ldots \\
-1 & -1 & -1 & \ldots & p^{\mathrm{t}}-1
\end{array}\right)_{p^{\mathrm{t} \times p^{\mathrm{t}}}}
$$

We now proceed to find the spectrum of $L$ for every prime $p$. The characteristic polynomial of $L$ is given by $\Lambda(x)=\operatorname{det}(x \mathrm{I}-\mathrm{L})$.

Apply the row operation $R_{1} \rightarrow \sum_{i=1}^{p^{t}} R_{i}$ and take $x$ common from 1 st row, we get

$$
\Lambda(x)=x \operatorname{det}\left(\begin{array}{ccccc}
1 & 1 & 1 & \ldots & 1 \\
1 & x-p^{t}+1 & 1 & \ldots & 1 \\
1 & 1 & x-p^{t}+1 & \ldots & 1 \\
\ldots & \ldots & \ldots & \ldots & \ldots \\
1 & 1 & 1 & \ldots & x-p^{t}+1
\end{array}\right)_{p^{t} \times p^{t}}
$$

Now we apply row operations $R_{i} \rightarrow R_{i}-R_{1}, \forall i=2,3, \cdots, p^{t}$, we get

$$
\Lambda(x)=x \operatorname{det}\left(\begin{array}{ccccc}
1 & 1 & 1 & \cdots & 1 \\
0 & x-p^{t} & 0 & \cdots & 0 \\
0 & 0 & x-p^{t} & \cdots & 0 \\
\cdots & \cdots & \cdots & \cdots & \cdots \\
0 & 0 & 0 & \cdots & x-p^{t}
\end{array}\right)_{p^{t} \times p^{t}}
$$

Thus, we have $\Lambda(x)=x\left(x-p^{t}\right)^{p^{t}-1}$. Hence the eigenvalues of $L$ are 0 with multiplicity 1 and $p^{t}$ with multiplicity $p^{t}-1$.

Theorem 4.3. Laplacian spectrum of group $\mathbb{Z}_{\mathfrak{p}^{\alpha_{1}}} \times \mathbb{Z}_{\mathfrak{p}^{\alpha_{2}}} \times \cdots \times \mathbb{Z}_{\mathbf{p}^{\alpha_{n}}}$ where $\mathrm{n} \geqslant 1$ are 0 with multiplicity 1 , $\mathrm{p}^{\alpha_{1}+\alpha_{2}+\cdots+\alpha_{n}}$ with multiplicity $\mathrm{p}^{\mathrm{n}}$ and $\mathrm{p}^{\mathrm{n}}$ with multiplicity $\mathrm{p}^{\alpha_{1}+\alpha_{2}+\cdots+\alpha_{n}}-\mathrm{p}^{\mathrm{n}}-1$ where $\mathrm{p}$ is a prime and $\max \left(\alpha_{1}, \alpha_{2}, \cdots, \alpha_{n}\right) \geqslant 2$.

Proof. We now proceed to find the Laplacian spectrum of group $\mathbb{Z}_{\mathfrak{p}^{\alpha_{1}}} \times \mathbb{Z}_{\mathrm{p}^{\alpha_{2}}} \times \cdots \times \mathbb{Z}_{\mathbf{p}^{\alpha_{n}}}$ and denoted by L.

The rows and columns of the matrix $L$ have been indexed in the following ways: We start with the zero element [0] of $\mathbb{Z}_{p^{\alpha_{1}}} \times \mathbb{Z}_{p^{\alpha_{2}}} \times \cdots \times \mathbb{Z}_{p^{\alpha_{n}}}$. We then list the all $p^{n}-1$ elements of order $p$ from group $\mathbb{Z}_{\mathfrak{p}^{\alpha_{1}}} \times \mathbb{Z}_{\mathfrak{p}^{\alpha_{2}}} \times \cdots \times \mathbb{Z}_{\mathfrak{p}^{\alpha_{n}}}$. We then list the remaining elements of $\mathbb{Z}_{\mathfrak{p}^{\alpha_{1}}} \times \mathbb{Z}_{\mathfrak{p}^{\alpha_{2}}} \times \cdots \times \mathbb{Z}_{\mathfrak{p}^{\alpha_{n}}}$. Using the above indexing the matrix of $L$ takes the following form:

$$
L=\left(\begin{array}{cc}
A & C \\
C^{\top} & B
\end{array}\right)
$$


where

$$
A=\left(\begin{array}{ccccc}
p^{\alpha_{1}+\alpha_{2}+\ldots+\alpha_{n}}-1 & -1 & -1 & \ldots & -1 \\
-1 & p^{\alpha_{1}+\alpha_{2}+\ldots+\alpha_{n}}-1 & -1 & \ldots & -1 \\
-1 & -1 & p^{\alpha_{1}+\alpha_{2}+\ldots+\alpha_{n}}-1 & \ldots & -1 \\
\ldots & \ldots & \ldots & \ldots & \ldots \\
-1 & -1 & -1 & \ldots & p^{\alpha_{1}+\alpha_{2}+\ldots+\alpha_{n}}-1
\end{array}\right)_{p^{n} \times p^{n}} .
$$

$C$ is a $p^{n} \times p^{\alpha_{1}+\alpha_{2}+\cdots+\alpha_{n}}-p^{n}$ matrix whose all entries are -1 and $B=p \mathrm{p}_{p^{\alpha_{1}+\alpha_{2}+\cdots+\alpha_{n}}-p^{n}}$. By using Theorem 4.1, we get Laplacian Spectrum of L as follows:

0 with multiplicity $1, p^{\alpha_{1}+\alpha_{2}+\cdots+\alpha_{n}}$ with multiplicity $p^{n}$ and $p^{n}$ with multiplicity $p^{\alpha_{1}+\alpha_{2}+\cdots+\alpha_{n}}-$ $p^{n}-1$.

Theorem 4.4. Laplacian spectrum of group $\mathbb{Z}_{\mathrm{p}}{ }^{\mathrm{t}} \times \mathbb{Z}_{\mathrm{q}}{ }^{\mathrm{s}}$ are 0 with multiplicity $1, \mathrm{p}^{\mathrm{t}} \mathrm{q}^{\mathrm{s}}$ with multiplicity $\mathrm{p}^{\mathrm{t}}+$ $\mathrm{q}^{\mathrm{s}}-1$ and $\mathrm{p}^{\mathrm{t}}+\mathrm{q}^{\mathrm{s}}-1$ with multiplicity $\mathrm{p}^{\mathrm{t}} \mathrm{q}^{\mathrm{s}}-\mathrm{p}^{\mathrm{t}}-\mathrm{q}^{\mathrm{s}}$ where $\mathrm{p}$ and $\mathrm{q}$ are distinct primes and $\mathrm{s}, \mathrm{t} \geqslant 1$.

Proof. We now proceed to find the Laplacian spectrum of group $\mathbb{Z}_{\mathrm{p}}{ }^{\mathrm{t}} \times \mathbb{Z}_{\mathrm{q}}{ }^{\mathrm{s}}$ and denoted by L. The rows and columns of the matrix $L$ have been indexed in the following ways: We start with the zero element $[0]$ of $\mathbb{Z}_{p}{ }^{t} \times \mathbb{Z}_{q}{ }^{s}$. We then list the all $p^{t}-1$ elements of order $p$ and $q^{s}-1$ elements of order $q$ from group $\mathbb{Z}_{p}{ }^{t} \times \mathbb{Z}_{q}{ }^{s}$. We then list the remaining elements of $\mathbb{Z}_{p}{ }^{t} \times \mathbb{Z}_{q}{ }^{s}$. Using the above indexing the matrix of $L$ takes the following form:

$$
L=\left(\begin{array}{cc}
A & C \\
C^{\top} & B
\end{array}\right)
$$

where

$$
A=\left(\begin{array}{ccccc}
p^{t} q^{s}-1 & -1 & -1 & \ldots & -1 \\
-1 & p^{t} q^{s}-1 & -1 & \ldots & -1 \\
-1 & -1 & p^{t} q^{s}-1 & \ldots & -1 \\
\ldots & \ldots & \ldots & \ldots & \ldots \\
-1 & -1 & -1 & \ldots & p^{t} q^{s}-1
\end{array}\right)_{p^{t}+q^{s}-1 \times p^{t}+q^{s}-1}
$$

$C$ is a $p^{t}+q^{s}-1 \times p^{t} q^{s}-p^{t}-q^{s}+1$ matrix whose all entries are -1 and $B=\left(p^{t}+q^{s}-1\right) I_{p^{t} q^{s}-p^{t}-q^{s}+1}$. By using Theorem 4.1, we get Laplacian spectrum of $L$ as follows: $q^{s}$

0 with multiplicity $1, p^{t} q^{s}$ with multiplicity $p^{t}+q^{s}-1$ and $p^{t}+q^{s}-1$ with multiplicity $p^{t} q^{s}-p^{t}-$

Theorem 4.5. Laplacian spectrum of group $\mathrm{D}_{\mathrm{p}^{n}}$ are 0 with multiplicity $1,2 \mathrm{p}^{\mathrm{n}}$ with multiplicity $\mathrm{p}+\mathrm{p}^{\mathrm{n}}$ and $\mathrm{p}+\mathrm{p}^{\mathrm{n}}$ with multiplicity $\mathrm{p}^{\mathrm{n}}-\mathrm{p}-1$ with condition that if $\mathrm{p}$ is a odd prime, then $\mathrm{n} \geqslant 1$ and if $\mathrm{p}$ is even prime than $n \geqslant 2$.

Proof. We now proceed to find the Laplacian spectrum of group $D_{p^{n}}$ and denoted by L. The rows and columns of the matrix $L$ have been indexed in the following ways: We start with the zero element [0] of $D_{p^{n}}$. We then list the all $p-1$ elements of order $p$ and $p^{n}$ elements of order 2 from group $D_{p^{n}}$. We then list the remaining elements of $D_{p^{n}}$. Using the above indexing the matrix of $L$ takes the following form:

$$
L=\left(\begin{array}{cc}
A & C \\
C^{\top} & B
\end{array}\right)
$$

where

$$
A=\left(\begin{array}{ccccc}
2 p^{n}-1 & -1 & -1 & \ldots & -1 \\
-1 & 2 p^{n}-1 & -1 & \ldots & -1 \\
-1 & -1 & 2 p^{n}-1 & \ldots & -1 \\
\cdots & \cdots & \cdots & \ldots & \ldots \\
-1 & -1 & -1 & \ldots & 2 p^{n}-1
\end{array}\right)_{p+p^{n} \times p+p^{n}}
$$


$C$ is a $p+p^{n} \times p^{n}-p$ matrix whose all entries are -1 and $B=\left(p+p^{n}\right) I_{p^{n}-p}$. By using Theorem 4.1, we get Laplacian spectrum of $L$ as follows:

0 with multiplicity $1,2 p^{n}$ with multiplicity $p+p^{n}$ and $p+p^{n}$ with multiplicity $p^{n}-p-1$.

\section{References}

[1] J. Abawajy, A. Kelarev, M. Chowdhury, Power graphs: a survey, Electron. J. Graph Theory Appl. (EJGTA), 1 (2013), 125-147. 1

[2] S. Banerjee, On a New Graph defined on the order of elements of a Finite Group, arXiv, 2019 (2019), 14 pages. 1

[3] F. Budden, Cayley graphs for some well-known groups, Math. Gaz., 69 (1985), 271-278. 1

[4] I. Chakrabarty, S. Ghosh, M. K. Sen, Undirected power graphs of semigroups, Semigroup Forum, 78 (2009), 410-426. 1

[5] K. Goebel, W. A. Kirk, A fixed point theorem for asymptotically nonexpansive mappings, Proc. Amer. Math. Soc., 35 (1972), 171-174. 1

[6] R. A. Horn, C. R. Johnson, Matrix analysis, Cambridge University Press, Cambridge, (2013).

[7] A. V. Kelarev, S. J. Quinn, A Combinatorial property and power graphs of Groups, Contributions to general algebra, 12 (1999), 229-235. 1

[8] X. Ma, H. Wei, L. Yang, The coprime graph of a group, Int. J. Group Theory, 3 (2014), 13-23. 1

[9] R. P. Panda, K. V. Krishna, On the minimum degree, edge-connectivity and connectivity of power graphs of finite groups, Comm. Algebra, 46 (2018), 3182-3197. 1

[10] R. P. Panda, K. L. Patra, B. K. Sahoo, On the minimum degree of the power graph of a finite cyclic group, J. Algebra Appl., 2020 (2020), 15 pages. 1

[11] A. Sehgal, S. N. Singh, The degree of a vertex in the power graph of a finite abelian group, arXiv, 2019 (2019), 7 pages. 1 